\title{
Low Energy (Anti)atoms for Precision Tests of Basic Physics
}

\author{
D.M. Silveira, O. Pereira, M. Veloso, and Claudio L. Cesar \\ Instituto de Física \\ Universidade Federal do Rio de Janeiro \\ 21945-970, Rio de Janeiro, RJ, Brazil
}

Received on 23 March, 2001

\begin{abstract}
Recent advances in techniques to manipulate and study, with high precision, atomic hydrogen, from one hand, and successful trapping schemes for positrons and antiprotons, from the other hand, have encouraged the pursuit of experiments to test CPT violation and the Weak Equivalence Principle (WEP) through the comparison of hydrogen and antihydrogen. A description of the hydrogen trap and laser system being built in Rio, to trap and perform high resolution spectroscopy on cold hydrogen, is presented along with a discussion on the techniques and experimental system being implemented by the ATHENA collaboration at CERN to produce cold antihydrogen. A new technique to make a cold antihydrogen beam is proposed.
\end{abstract}

\section{Introduction}

CPT invariance can be derived from very general principles of relativistic quantum field theories and its conservation theorem has been proved in flat space-time[1]. The combined operation of charge conjugation (C), space reflection $(\mathrm{P})$ and time reversal $(\mathrm{T})$ should represent an exact symmetry of nature. By testing CPT invariance, one is testing the correctness of the description of the microscopic world in terms of the exhisting field theory; one is testing the Standard Model [2]. In what concerns antimatter, it predicts that particles and antiparticles shall have equal masses and lifetimes, as well as equal (but opposite) charges and magnetic moments. For (anti)atomic systems the predictions are that the levels' energies and lifetimes should be the same for the conjugate species. To date, no CPT violation has been experimentally observed. Precision tests of CPT violation include measurements of the difference between the magnetic moments of the electron and positron and the difference between the charge-to-mass ratio of the proton and antiproton. Nevertheless, the most precise test $\left(10^{-18}\right.$ accuracy)[3], though model dependent, comes from the comparison between the mass of the neutral kaon and antikaon (see Fig. 1).

Precision measurements with the simplest antiatom that could be produced at low energies would represent a more vigorous, model-independent test of CPT invariance. Moreover, the availability of these slow antiatoms would prompt experiments to compare the gravitational accelerations of hydrogen and antihydrogen, a direct test of the Weak Equivalence Principle
(WEP): for given initial conditions, neglecting spin effects, equal bodies follow the same trajectories [4]. If this principle does not hold under experimental verification, General Relativity will have to be revised. A direct measurement of the gravitational acceleration of hydrogen and antihydrogen would address an old and renewed question on the possibility of antigravity [5] or a WEP breakdown[6], an issue that still lacks experimental observation. The challenge is to produce suitably cold antihydrogen.

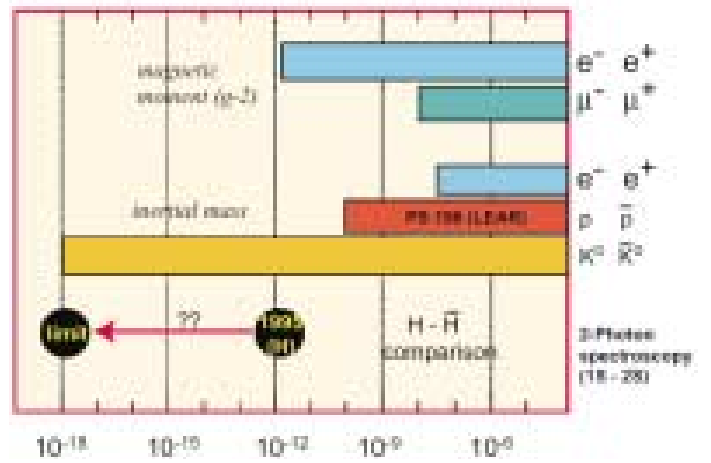

Figure 1. Comparison between precision tests of CPT invariance. See text for discussion.

Antihydrogen is believed to have been first produced and detected in 1996[2], in a team experiment held at CERN's LEAR (Low Energy Antiproton Ring). The basic idea is that an antiproton passing through the Coulomb field of a nucleus will create an electronpositron pair. Ocasionally the antiproton will capture 
a positron from the produced pair and form a fast moving antihydrogen atom. In the CERN experiment, the xenon atoms (the target) were shot from a gas-jet nozzle across the path of the antiprotons as they circulated around LEAR. Antihydrogen was detected through the annihilation of the antiatoms' positrons with the electrons of a silicon target, with the emission of a characteristic photon pair detected by a set of surrounding $\mathrm{NaI}$ calorimeters. Additional measurements down the line on the stripped antiprotons eliminate all but antihydrogen events. Though it was nice to observe antihydrogen for the first time, very little could be done on the study of its properties as the few antiatoms produced (nine counts could be assigned to antihydrogen) travelled a few nanoseconds before annihilation. Later on, antihydrogen was produced in larger amounts at Fermilab[7]. For a comprehensive study of its properties, antihydrogen has to be produced with much less speed and in much greater numbers.

This is the challenge posed to the ATHENA collaboration[4]: the production of antihydrogen in a novel way so as to get very low energy antiatoms suitable for high precision measurements. All the ingredients to form antihydrogen are at hand. Since the early 80's steady progress has been made in cooling and trapping neutral atoms; in particular, ultracold gaseous samples of spin-polarized atomic hydrogen have been studied with high precision through twophoton Doppler-free laser spectroscopy on the 1S-2S transition[8]. Recent advances in capture, cooling and trapping of positrons and antiprotons make it feasible to propose recombination schemes with the aim of forming cold antihydrogen.

In this paper we address some of the experimental issues involved in this research, its current status and propose a new method to generate a cold antihydrogen beam. In section II we present the current status of trapped hydrogen research and our apparatus using the buffer gas loading technique and the laser system for spectroscopy. In section III we discuss the ATHENA approach and current status on antiprotons and positrons trapping and recombination schemes for antihydrogen production. Section IV discusses the integration of these aspects and physics experiments, concluding in section $\mathrm{V}$.

\section{Trapped atomic hydrogen and laser spectroscopy}

Bose-Einstein condensation (BEC) was the main driving force behind research with cold hydrogen, as it had been predicted that spin-polarized hydrogen would remain in atomic form down to $\mathrm{T}=0 \mathrm{~K}[9]$. The first magnetic trapping of atomic hydrogen was done at MIT in 1987[10] using an evacuated cell cooled by a ${ }^{3} \mathrm{He}^{-}{ }^{4} \mathrm{He}$ dilution refrigerator and covered with superfluid helium within an inhomogeneous magnetic field. Molecular hydrogen, frozen on the walls, is desorbed and dissociated by a RF discharge. The resulting hydrogen atoms can lose energy and bounce off the weakly binding helium film, on the cell walls. The slow atoms are then magnetically trapped through the interaction of its magnetic dipole moment $\mu$ with the external inhomogeneous field B. In the presence of external fields, the atoms' energy levels split into hyperfine levels whose energy increase or decrease with the applied field. Since Maxwell's equations forbid the creation of a point of field maximum in a source-free region, one can trap atoms whose energy increase with increasing field in a point of field minimum; atoms in such states are called 'low-field seekers'.

That same year the group at MIT was able to test the proposal[11] of forced evaporative cooling achieving temperatures as low as $1 \mathrm{mK}[12]$. This technique eliminates the high energy atoms, which are continuosly being generated by collisions within the thermal sample. The removal of excess energy causes the sample to thermalize at ever lowering temperatures. Typically one loses one order of magnitude in atom number while gaining two orders of magnitude in the decrease of the temperature. A new and larger trap was built[13] and the MIT group got to temperatures as cold as $100 \mathrm{mK}$ and densities as high as $5 \times 10^{13} \mathrm{~cm}^{-3}$. Bose-Einstein condensation could not be seen in the system due to the lack of a good detector. It was then invested a large effort in developing the laser and optical system for the detection of the trapped atoms using the $1 \mathrm{~S}-2 \mathrm{~S}$ transition, to be used as a detection scheme for BEC and for the interest of spectroscopy on this fundamental atom.

A few years later, in 1995, the MIT group[8] was able to perform laser spectroscopy on the trapped atoms at $400 \mu \mathrm{K}$ achieving a record high resolution of 2 parts in $10^{12}$ at the time. In a recent publication, Cesar and Kleppner[14] showed how, in this low energy regime, one can avoid time-of-flight broadening, which, along with the second-order Doppler effect, is the dominant broadening mechanism of today's state-of-the-art hydrogen atomic beam[15]. But either as a frequency standard or as a portable reference trap for comparisons with antihydrogen, the MIT trap is too complicated and costly to be a viable option. Other trapping strategies have to be developed to make it less complex and more reliable.

One of the most important parameters of a magnetic trap is its depth, the difference between the potential (or the field) on the edge and on the bottom of the trap. Despite the amazing development of superconductors and permanent magnets technology, it remains a challenge to create traps deeper than 3-4 T, which corresponds to $2.5 \mathrm{~K}$ for a $1 \mu_{B}$-dipole moment atom, 
like hydrogen. It is clear that the atoms that are to be trapped must undergo some kind of pre-cooling so that its energy is lowered to a point where the gaseous sample can be confined by the magnetic trap. This has been made basically by either of two techniques: laser cooling - which works fine for alcaline atoms with a simple scheme of energy levels and laser-friendly, easily accessible transitions - or thermalization with a thin superfluid liquid ${ }^{4} \mathrm{He}$ film, which is well suited only for hydrogen. Despite huge efforts in extending the range of applicability of optical techniques, most of the elements of the periodic table (as well as molecules) remain untouchable by those techniques.

\section{A. Buffer gas loading}

Buffer gas loading of magnetic traps is an alternative technique that proved to be widely applicable. Proposed[16] and demonstrated[17] by John Doyle's group at Harvard, it was employed to magnetically trap large amounts of molecules[18], a result never achieved before. The basic idea is to cool the gaseous sample through elastic collisions with the cold buffer gas in the regime of large Knudsen's number, i.e., the mean free path much shorter than the cell dimensions. The buffer gas - typically ${ }^{3} \mathrm{He}$ or ${ }^{4} \mathrm{He}$ which have reasonably high vapour pressure in the sub-kelvin region - is maintained in a cryogenic cell at temperatures below $1 \mathrm{~K}$. In Doyle's trap this temperature is obtained through a thermal link between the experimental cell and the mixing chamber of a dilution refrigerator.

Atoms can be introduced into the cell by either a RF discharge that desorbs atoms frozen on the cell walls or by laser ablation of a solid lump. For hydrogen, a low temperature RF pulsed discharge would be preferred, since the molecular hydrogen snow is spaced thorough the cell walls. Atomic fluxes of $10^{12} \mathrm{~s}^{-1}$ and temperatures no greater than $10^{3} \mathrm{~K}$ are typical values for such discharges. The buffer gas serves the purpose of reducing the atoms' kinetic energy while keeping them away from the cell walls where they would stick. Since the technique relies only on elastic collisions and not on cyclic optical transitions it is of general application and can be employed to trap large amounts of more than $70 \%$ of the atoms (those with $\mu \geq 1 \mu_{B}$ ), as well as molecules. Its efficiency depends on the ratio of the magnetic energy depth to the sample's initial loading temperature.

Although the dilution refrigerator allows the cell to be kept at temperatures as low as $100 \mathrm{mK}$, it is expensive, fairly large and it is not an user-friendly equipment. Pumping on liquid ${ }^{4} \mathrm{He}$ one can reach beyond the superfluid transition at $2.2 \mathrm{~K}$, or $\lambda$-point. A typical $1 \mathrm{~K}$ pot cryostat regulates its temperature at $1.3 \mathrm{~K}$, where the vapour pressure is enough to allow efficient removal of latent heat of evaporation to compensate for the heat conduted by the superfluid from hotter areas and for cooling the cell. Estimating the efficiency of buffer gas loading in a $1 \mathrm{~K}$ pot environment one finds a lower performance than with the dilution refrigerator but still adequate for the second phase of the ATHENA experiment, where the trapped hydrogen will serve as a reference for spectroscopic comparison with the antihydrogen. The added benefits of the $1 \mathrm{~K}$ pot are in cost, complexity and turn around time.

\section{B. The apparatus in Rio}

In Rio we are setting up an apparatus with a $1 \mathrm{~K}$ pot to perform buffer gas loading and magnetic trapping of paramagnetic atomic and molecular species including atomic hydrogen - with the aim of further cooling the trapped samples through forced evaporative cooling[11]. The cell and the $1 \mathrm{~K}$ pot are contained inside the magnetic trap consisting of a spherical quadrupole type: two superconducting NbTi coils in an anti-Helmholtz arrangement. Field is zero at the trap center and rises in all directions; the trap center is a $3 \mathrm{D}$ magnetic field minimum and it's around this point that the atoms remain trapped. The magnet cask is a titanium single piece, designed to withstand the brutal repulsion forces of a pair of $100 \mathrm{~A}$ opposing coils, and it is completely immersed in liquid helium. The whole experiment (cell, $1 \mathrm{~K}$ pot, superconducting magnet, liquid helium reservoir) is contained in a specially designed cryostat as depicted in Fig. II. It features a liquid nitrogen reservoir - to reduce thermal conduction to the liquid helium - and a highly conducting, low emissivity metallic shield thermally anchored at $77 \mathrm{~K}$, to protect the helium environment from the $300 \mathrm{~K}$ black-body radiation. Optical access to the cell is through a series of optical windows.

Buffer-gas loaded, magnetically trapped atomic hydrogen is also very interesting from the metrology point-of-view. Besides building a system for direct comparison of the 1S-2S transition in hydrogen and antihydrogen, we plan to build another system to be used as an optical frequency standard based on this same transition. To date, the most precise optical frequency measurement was made in hydrogen atomic beams through phase-coherent comparison to a cold Cs atomic fountain clock [15]. The trapped hydrogen-based system has potential for much higher resolution than the atomic beam-based spectrometer at Garching.

Magnetic trapping and forced evaporative cooling of atomic species other than hydrogen or alcaline-metal as well as molecules - are also being pursued in our laboratory. Our first attempts shall employ high magnetic moment species, like europium. For most species, it is reported that laser ablation is much more appropriate than RF discharges [17]; for this we use a 
pulsed Nd:YAG laser source. After loading and trapping, forced evaporative cooling shall be performed to reduce the sample's temperature from $1 \mathrm{~K}$ to the $\mu \mathrm{K}$ range. We are specially interested in sympathetic evaporation, where cooling of one species for which evaporation works fine leads to cooling of another (simultaneously trapped) species which is not suited for evaporation.

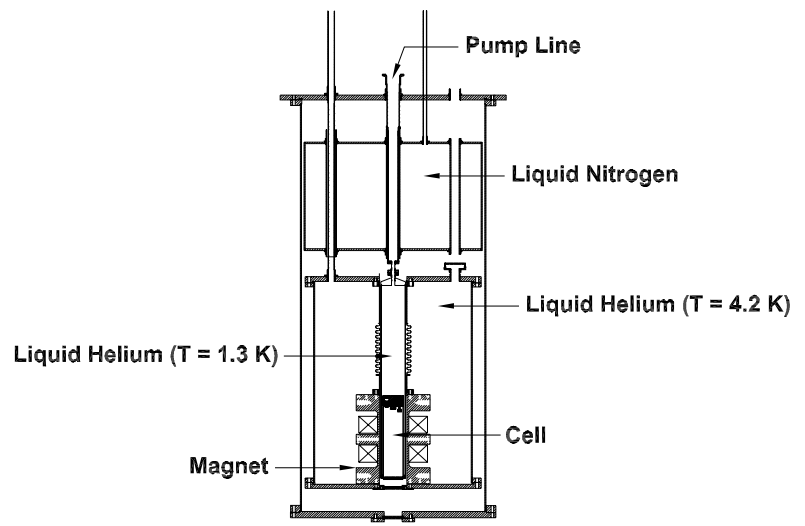

Figure 2. Depiction of the $1 \mathrm{~K}$ cryostat. See text for discussion.

\section{The laser system for spectroscopy}

Atomic hydrogen has proved to be a great laboratory for tests of the most basic theories of Physics. Recent spectroscopic measurements on the 1S-2S transition has had implications on the measurement of the Lamb shift[19] and on corrections to QED[20]. This transition is accessed with laser radiation at $243 \mathrm{~nm}$ which can be produced by frequency doubling from a dye laser at $486 \mathrm{~nm}$ as is done at MIT and will be pursued at ATHENA, or by frequency quadruplicating from a Ti:saphire laser, as we are implementing in Rio.

The choice for this laser system was dictated by the need of flexibility to access different atomic and molecular species in our own experimental program. It consists of a comercial (Coherent, Inc.) Argon Ion laser whose output of up to $28 \mathrm{~W}$ is used to pump a Coherent 899-21 Titanium:saphire laser which has its frequency doubled twice. The Ti:saphire laser oscilates in the range of $700 \mathrm{~nm}$ to $1100 \mathrm{~nm}$. Typical peak power at $15 \mathrm{~W}$ of pump power is $0.6 \mathrm{~W}$, CW single mode, at $972 \mathrm{~nm}$. For the first doubling stage we employ an external enhancement cavity and a $\mathrm{KNbO}_{3}$ crystal, as schematically shown in Fig. 3. Potassium Niobate has optical transparency ranging from 400 to $4500 \mathrm{~nm}$. It presents the largest non-linear coefficients of all comercially available inorganic material $\left(\mathrm{d}_{31}=-15.8 \mathrm{pm} / \mathrm{V}\right.$, $\mathrm{d}_{32}=-18.3 \mathrm{pm} / \mathrm{V}$ ) and, with its negative biaxial structure, is suitable for a wide variety of temperature-tuned type I non-critical phase-matching (NCPM) and type II, angle tuned, phase-matching configurations. It essentially allows one to cover SHG from $860 \mathrm{~nm}$ to well beyond the Nd:YAG wavelength $(1.064 \mathrm{~mm})$. We were already generating blue light while scanning the cavity and we will try a new proposed method to lock the doubling cavity[21] (see Fig. 3) after the laser's "intracavity assembly" is repaired at the manufacturer. This laser system will serve for studying other atomic and molecular species as well.

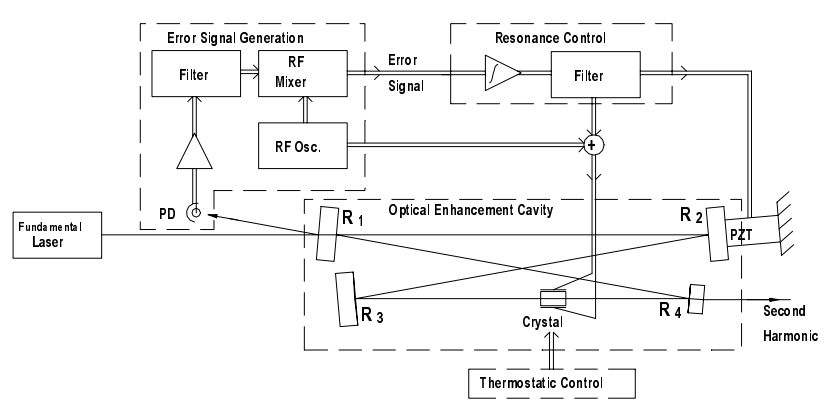

Figure 3. Schematics of the first optical frequency doubling cavity, showing the lock scheme. The crystal at use is $\mathrm{KNbO}_{3}$.

The unusually long lifetime of the metastable $2 \mathrm{~S}$ level $(122 \mathrm{~ms})$ makes it possible, at least theoretically, for one to measure the 1S-2S energy difference with a $10^{-18}$ accuracy, provided the (anti)atoms are trapped and cold. The excitation of the 1S-2S transition is performed by two-photon absorption. The $2 \mathrm{~S}$ state can be quenched by a small electric field which causes a mixing with the $2 \mathrm{P}$ state resulting in the emission of a Lyman $\alpha$ photon, which is typically detected in a microchannel plate followed by an amplifying stage and counting system. For the antihydrogen one can use different schemes relying on the annihilation signal. As proposed by P. Bowe and J. Hangst[22] one could use photoionization of the excited atom and electrically reflect back the antiparticles while letting the non-excited antiatoms go straight onto an annihilation wall. Or, in the case of trapped antihydrogen, the $\operatorname{Lyman}_{\alpha}$ decay also leads, sometimes, to a change of spin state, causing the atoms to be expelled from the trap and the anihilation signal to be seen, as proposed by one of us[23].

\section{Towards cold antihydrogen}

In the last years researchers working at accelerators have succeded in developing new methods for manipulating antiparticles. Antiprotons and positrons have been captured, slowed and stored for long times in electromagnetic traps; techniques for creating antimatter beams were also devised. Some of these methods are employed in the ATHENA experiment to pursuit the formation of atomic hydrogen. 
In CERN's LEAR, antiprotons have been captured and cooled to meV energies by suitable methods. The same schemes are used with CERN's new antiproton machine, the Antiproton Decelerator (AD). The Antiproton Decelerator (AD) started delivering antiprotons to the experiment by mid of 2000. Soon after the ATHENA team reported capture of antiprotons. After being slowed down in a beam counter, the antiprotons pass a foil degrader with adjustable effective thickness (a split Si photodiode - for reference and centering of the beam) and enter the appropriate Penning trap, which had been previously filled with electrons. As antiprotons oscillate back and forth in the trap they loose their energy through Coulomb collisions with the electron cloud. This capture process can be repeated several times without losing trapped antiprotons, thus enhancing the total number of antiprotons to be used in the experiment. With the dump signal from the trap the foil thickness could be varied for optimization. The vacuum conditions in this first run of ATHENA was not in a great shape and the lifetime of the antiparticles are directly affected by anihilation with the background gas. Typically the sample could last for hours in a ideal vacuum conditions. In the experimental condition shown the sample lifetime was reduced to $10 \mathrm{~s}$. Obviously this can be improved and one of the major factors is the temperature of the cold nose of the experiment which was high and this problem is being addressed for the 2001 run.

A second important step towards the goals of ATHENA is the production of slow positrons. These low energy positrons (kinetic energies in the $\mathrm{MeV}$ range) are available in a controlled way either from radioactive sources or from pair production from bremsstrahlung. Their energies can be further reduced upon penetration into a moderator. Low energy positrons are readily slowed to the thermal level and a fraction of it is spontaneously emitted in vacuum either as free positrons or as positronium. Positron accumulation for ATHENA has been achieved by the Swansea group with the newly built positron accumulator following a design by C. Surko's group at U.C. San Diego. This design uses a ${ }^{22} \mathrm{Na}$ source onto which surface is deposited a thin layer of solid neon (moderation efficiencies as high as $10^{-3}$ ), at cryogenic temperatures. This system provides the best demonstrated source of thermal positrons. After the source and following the magnetic field lines the positrons encounter different regions with a varying pressure of $\mathrm{N}_{2}$ serving as a thermalizing buffer gas and as a dissipation mechanism for loading the positron Penning trap. After initial trapping, buffer gas pressure is reduced to allow long storage times. As a typical value achieved in 2000, an excess of $4 \times 10^{6}$ positrons were trapped. The positrons couple radiatively (via cyclotron radiation) and inductively to the wall and they quickly thermalize with the walls temperature. This system will be improved by over an order of magnitude this year mainly by the use of a stronger source.

The third major step in the ATHENA experimental scheme is the transfer of the cold positrons to the recombination trap next to the antiproton trap. This is achieved by an electric kick on the positrons and the simultaneous openning of a vacuum valve and the pulsing of the "transfer magnet". While there was a signal clearly indicating the transfer of the positrons to the antiproton region, with an efficiency of about $25 \%$, there was no attempt to retrap these particles. This process is being pursued now at CERN.

A fourth major ingredient is the antiparticle anihilation detector. Built with a concentric layer of 192 CsI crystals whose scintilation signals are detected by a photodiode, and two layers of Si-strip detectors, this detector assembly, with its integrated electronics can work at liquid Nitrogen temperature and is able of providing vertex reconstruction for an anihilation event with $100 \mu \mathrm{m}$ radial resolution and $0.7 \mathrm{~mm}$ axial resolution. This detection system should provide the experiment with an unambiguos antihydrogen detection. The detector has been fully calibrated and no major improvement is required for this year's run.

The major milestone eagerly pursued by the ATHENA experiment in the next years is the formation of low-energy antihydrogen. There are a few schemes which can be pursued for this recombination and we still don't know of all the details on their outcome. Whatever recombination method is used, it must yield a sufficient number of antiatoms for spectroscopy, preferably at low temperatures $(\mathrm{T}>1 \mathrm{~K}$ should be avoided since this would require prohibitevely high magnetic well depths). These atoms should also be produced in the ground state or in a low-lying excited state and the whole process should happen within a short time period.

The basic idea from the ATHENA team is to shoot the antiprotons in a controllable fashion on the positron cloud in such a way that they would stop in the middle of it (see Fig. III) . They would then drift slowly through the positron cloud, in a non-trapped configuration, until they recombine or scape and are recycled back to the antiproton trap. This procedure could, in principle, be repeated many times. This method will certainly ensure the mixing of the two species at the lowest possible relative velocities therefore garanteeing the best possible recombination rate for a spontaneous recombination process. To form a bound state of positron and antiproton starting from free particles, momentum and energy balance requires the presence of a third body. Spontaneous recombination can thus be described as: 


$$
e^{+}+\bar{p} \rightarrow \bar{H}+h \nu
$$

There are a few unknowns in this scheme which will be studied experimentally. The first is how efficiently can one cycle back the antiprotons and efficiently keep them cold. The second is how much of the magnetron motion from the positron cloud will get transfered to the antiprotons and therefore get transfered to the formed antihydrogen. In principle, the rotating energy of the positron plasma is much larger than its quoted temperature. If all this energy gets transferred into kinetic energy of the recombined antihydrogen the chance of ever capturing enough of these in a magnetic trap is minimal, due to the low magnetic energy. On the other hand, if a very small fraction, or none, of this energy is transfered, the antihydrogen will come out of the positron cloud in all directions but with small energy. This is not an ideal situation for any imaginable experiment that will have to rely on long interaction times and good detection collection efficiency. A much better situation would be to have an atomic beam. In view of this desire we have proposed a simple scheme to generate an antiatomic beam as we now explain.

\section{A. Forming an atomic beam}

With many centimeters of trap dimension we have enough space to allow the positron cloud to travel with a constant speed while the antiprotons are coming towards it. The most probable situation for recombination will be when the antiprotons are at rest in the moving positron cloud frame. Once the neutral antiatom is formed it is not dragged by the positrons anymore. The positrons are electrically stopped upstream while the antihydrogen may continue travelling as a cold atomic beam. Once the non-recombined antiprotons are recaptured back at its original trap the procedure can be repeated again. One could expect, therefore, a pulsed atomic beam operation. If one considers a $10 \mathrm{~K}$ energy atomic beam for a laser excitation experiment, for example, the typical speed required for the positron cloud and the outgoing antihydrogen atoms is about $400 \mathrm{~m} / \mathrm{s}$. If the whole process is to last a few miliseconds, the space required for the positron cloud to move is about half a meter. The controlled movement of the positron cloud could be made adiabatically by the use of multiple electrodes and the control electronics is trivial to build since it is a slow system.

If such a beam can be made, a few imediate experiments come to mind. First we could easily study the angular dispersion of the beam just by looking at the anihilation signal at a far away wall closely surrounded by a detector. Secondly, by varying the speed of moving positron cloud and the length of time, one will certainly be able to estimate pretty well the energy distribution of the formed antihydrogen. Thirdly, knowing the energy distribution one can easily consider the feasibility of performing laser spectroscopy on the $1 \mathrm{~S}-2 \mathrm{~S}$, using the photoionization method mentioned above. Finally, one can also attempt to look for antigravity by the deviation of the cloud from the trajectory that would be initially followed by the positrons and antiprotons along the magnetic field lines, by simply turning off the reflection electric potential.

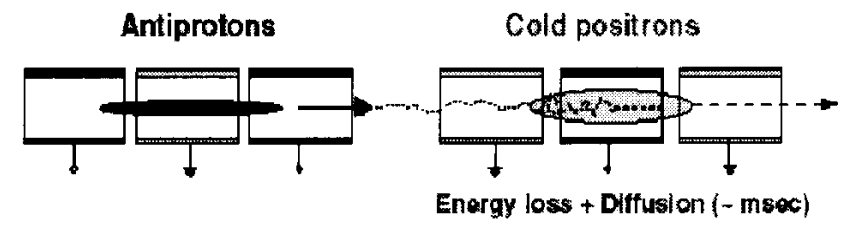

Figure 4. One of the possible recombination schemes to be used for the formation of antihydrogen.

\section{Physics with cold antihydro- gen}

As pointed out in the introduction, the main goal of the ATHENA experiment is to test CPT invariance as precisely as possible. This will be possible at a level of parts in $10^{10}$ as soon as ATHENA succeds in producing a cold atomic beam at the energy conditions discussed above and with the excitation and detection of a few hundred antiatoms. Higher precision is forseen in phase II, when the plan is to trap the cold antihydrogen. Later on, a cooling scheme will be pursued.

Antihydrogen atoms produced at low temperatures are very interesting for gravitational tests. The measurement of the gravitational properties of antihydrogen is a direct test of the Weak Equivalence Principle (WEP), the cornerstone of General Relativity. The most stringent WEP test to date was performed by comparing the accelerations of different types of matter[24], with a precision of 1 part in $10^{11}$, but no direct test of WEP has ever been performed using antimatter. A famous experiment[6] has measured directly the gravitational acceleration of electrons, but its results were found to be inconclusive because charged particles are very sensitive to stray electric fields. It has been suggested[5] that antimatter may behave differently from matter in a gravitational field. A direct test of WEP on antiparticles is of fundamental relevance, and antihydrogen is the ideal candidate: not only is it neutral but also much more massive than positrons. There are currently some proposals for experiments to measure the gravitational acceleration of antihydrogen from collaboration members[25]. It is clear 
that, if any of these experiments should ever be performed, it will not be as precise as CPT tests. Nevertheless, it is of fundamental importance to observe whether antimatter falls down with the same acceleration as matter or with a different acceleration or even if antimatter is repelled from ordinary matter; in this latter case, one could talk about antigravity.

\section{Conclusion}

In this paper we presented our contributions to the ATHENA experiment, which has been running for about one year. This collaboration is aimed at producing antihydrogen at low energies suitable for high precision tests of CPT invariance. Tests will rely on the comparison between the $1 \mathrm{~S}-2 \mathrm{~S}$ transition in hydrogen and antihydrogen, studied through Doppler-free laser spectroscopy. We also described the idea of using the buffer gas technique for loading hydrogen into magnetic traps using a simple ${ }^{4} \mathrm{He}$-pumped system. This system is under implementation in Rio, in order to make a portable reference hydrogen trap for comparison with antihydrogen. Theoretical implications of the experiment include not only CPT violation - a test of the Standard Model - but also direct tests of the gravitational coupling to antimatter, a test of the Weak Equivalence Principle, a cornerstone of General Relativity.

Just how well will ATHENA do in this comparison, is too early a question. Nature and our scientific resources should still have much to say about this very challenging and interesting experiment!

\section{Acknowledgements}

This research was in part supported by $\mathrm{CNPq}$ and PRONEX(Brazilian agencies), and physics discussions with the MIT ultracold hydrogen group, J. Doyle at Harvard, and with ATHENA collaboration members are thankfully acknowledged.

\section{References}

[1] J.J. Sakurai, Invariance Principles and Elementary Particles, Princeton University Press, Princeton (1964).

[2] G. Baur, G. Boero, S. Brauksiepe, A. Buzzo, W. Eyrich, R. Geyer, D. Grzonka, J. Hauffe, K. Kilian, M. Lo Vetere, M. Macri, M. Moosburger, R. Nellen, W. Oelert, S. Passaggio, A. Pozzo, K. Röhrich, K. Sachs, G. Schepers, T. Sefzick, R. S. Simon, R. Stratmann, F. Stinzing and M. Wolke, Phys. Lett. B 368, 251 (1996).

[3] B. Schwingenheuer, R.A. Briere, A.R. Barker, E. Cheu, L. K. Gibbons, D. A. Harris, G. Makoff, K. S. McFarland, A. Roodman, Y. W. Wah, B. Winstein, R. Winston, E. C. Swallow, G. J. Bock, R. Coleman, M. Crisler, J. Enagonio, R. Ford, Y. B. Hsiung, D. A.
Jensen, E. Ramberg, R. Tschirhart, and T. Yamanaka, E. M. Collins and G. D. Gollin, P. Gu, P. Haas, W. P. Hogan, S. K. Kim, J. N. Matthews, S. S. Myung, S. Schnetzer, S. V. Somalwar, G.B. Thomson and Y. Zou, Phys. Rev. Lett. 74, 4376 (1995).

[4] M.H. Holzscheiter, G. Bendiscioli, A. Bertin, G. Bollen, M. Bruschi, C. Cesar, M. Charlton, M. Corradini, D. DePedis, M. Doser, J. Eades, R. Fedele, X. Feng, F. Galluccio, T. Goldman, J.S. Hangst, R. Hayano, D. Horváth, R.J. Hughes, N.S.P. King, K. Kirsebom, H. Knudsen, V. Lagomarsino, R. Landua, G. Laricchia, R.A. Lewis, E. Lodi-Rizzini, M. Macri, G. Manuzio, U. Marconi, M.R. Masullo, J.P. Merrison, S.P. Moller, G.L. Morgan, M.M. Nieto, M. Piccinini, R. Poggiani, A. Rotondi, G. Rouleau, P. Salvini, N. Semprini-Cesari, G.A. Smith, C.M. Surko, G. Testera, G. Torelli, E. Uggerhoj, V.G. Vaccaro, L. Venturelli, A. Vitale, E. Widmann, T. Yamazaki, Y. Yamazaki, D. Zanello and A. Zoccoli, Hyp. Int., 109, 1 (1997).

[5] G. Chardin, Hyp. Interact. 109, 83 (1997).

[6] F.C. Witteborn and W.M. Fairbank, Phys. Rev. Lett. 19, 1049 (1967)

[7] G. Blanford, D.C. Christian, K. Gollwitzer, M. Mandelkern, C. T. Munger, J. Schultz and G. Zioulas, Phys. Rev. Lett. 80, 3037 (1998).

[8] C.L. Cesar, D. G. Fried, T. C. Killian, A. D. Polcyn, J. C. Sandberg, I. A. Yu, T. J. Greytak, D. Kleppner and J. M. Doyle, Phys. Rev. Lett. 77, 255 (1996).

[9] W. Stwalley and L. Nosanow, Phys. Rev. Lett. 36, 910 (1976).

[10] H.F. Hess, G.P. Kochanski, J.M. Doyle, N. Masuhara, D. Kleppner and T. J. Greytak, Phys. Rev. Lett. 59, 672 (1987).

[11] H.F. Hess, Phys. Rev. B 34, 3476 (1986).

[12] N. Masuhara, J.M. Doyle, J.C. Sandberg, D. Kleppner, T. J. Greytak, H. F. Hess and G. P. Kochansky, Phys. Rev. Lett. 61, 935 (1988).

[13] J.M. Doyle, J.C. Sandberg, I.A. Yu, C.L. Cesar, D. Kleppner and T. J. Greytak, Phys. Rev. Lett. 67, 603 (1991).

[14] C.L. Cesar and D. Kleppner, Phys. Rev. A, 59, 4564 (1999).

[15] M. Niering, R. Holzwarth, J. Reichert, P. Pokasov, T. Udem, M. Weitz, T.W. Haensch, P. Lemonde, G. Santarelli, M. Abgrall, P. Laurent, C. Salomon and A. Clairon, Phys. Rev. Lett. 84, 5496 (2000).

[16] J.M. Doyle, B. Friedrich, J. Kim and D. Patterson, Phys. Rev. A 52, R2515 (1995).

[17] J. Kim, B. Friedrich, D.P. Katz, D. Patterson, J. D. Weinstein, R. deCarvalho and J. M. Doyle, Phys. Rev. Lett. 78, 3665 (1997); J. D. Weinstein, R. deCarvalho, J. Kim, D. Patterson, B. Friedrich and J. M. Doyle, Phys. Rev. A 57, R3173 (1998).

[18] J.D. Weinstein, R. deCarvalho, T. Guillet, B. Friedrich and J. M. Doyle, Nature 395, 148 (1998) 
[19] B. de Beauvoir, C. Schwob, O. Acef, L. Jozefowski, L. Hilico, F. Nez, L. Julien, A. Clairon and F. Biraben, Eur. Phys. J. D 12, 61 (2000)

[20] A. Huber, Th. Udem, B. Gross, J. Reichert, M. Kourogi, K. Pachucki, M. Weitz and T. W. Hõnsch, Phys. Ver. Lett. 80, 468 (1998).

[21] C.L. Cesar, accepted for publication in J. Opt. Soc. Am. B (2001).

[22] P. Bowe and J. Hangst, Scientific Collaboration Meet- ing (unpublished) (1999).

[23] C.L. Cesar, Scientific Collaboration Meeting (unpublished) (1999).

[24] Y. Su, B.R. Heckel, E.G. Adelberger, J.H. Gundlach, M. Harris, G. L. Smith and H. E. Swanson, Phys. Rev. D 50, 3614 (1994).

[25] C.L. Cesar, Hyp. Interact. 109, 293 (1997); R. Poggiani, Hyp. Interact. 109, 367 (1997). 